OPEN ACCESS

Edited by:

Ren Wei,

Leipzig University, Germany

Reviewed by:

Bo Yu,

Institute of Microbiology (CAS), China

Jan Baeyens,

University of Warwick

United Kingdom

${ }^{*}$ Correspondence:

Luo Liu

liuluo@mail.buct.edu.cn

Specialty section:

This article was submitted to Microbiotechnology, Ecotoxicology

and Bioremediation,

a section of the journal

Frontiers in Microbiology

Received: 07 February 2019

Accepted: 26 February 2019

Published: 12 March 2019

Citation:

Xu J, Cui Z, Nie K, Cao H, Jiang M, Xu H, Tan T and Liu L (2019)

A Quantum Mechanism Study of the C-C Bond Cleavage to Predict the Bio-Catalytic Polyethylene

Degradation

Front. Microbiol. 10:489. doi: 10.3389/fmicb.2019.00489

\section{A Quantum Mechanism Study of the C-C Bond Cleavage to Predict the Bio-Catalytic Polyethylene Degradation}

\author{
Junyu Xu' ${ }^{1}$, Ziheng Cui ${ }^{1}$, Kaili Nie ${ }^{1}$, Hao $\mathrm{Cao}^{2}$, Min Jiang ${ }^{3}$, Haijun $\mathrm{Xu}^{1}$, Tianwei $\operatorname{Tan}^{1}$ and \\ Luo Liu' ${ }^{1 *}$ \\ ${ }^{1}$ Beijing Key Laboratory of Bioprocess, Beijing University of Chemical Technology, Beijing, China, ${ }^{2}$ Laboratory \\ of Biomanufacturing and Food Engineering, Institute of Food Science and Technology, Chinese Academy of Agricultural \\ Sciences, Beijing, China, ${ }^{3}$ State Key Laboratory of Materials-Oriented Chemical Engineering, College of Biotechnology \\ and Pharmaceutical Engineering, Nanjing University of Technology, Nanjing, China
}

The growing amount of plastic solid waste (PSW) is a global concern. Despite increasing efforts to reduce the residual amounts of PSW to be disposed off through segregated collection and recycling, a considerable amount of PSW is still landfilled and the extent of PSW ocean pollution has become a worldwide issue. Particularly, polyethylene (PE) and polystyrene (PS) are considered as notably recalcitrant to biodegradation due to the carbon-carbon backbone that is highly resistant to enzymatic degradation via oxidative reactions. The present research investigated the catalytic mechanism of P450 monooxygenases by quantum mechanics to determine the bio-catalytic degradation of PE or PS. The findings indicated that the oxygenase-induced free radical transition caused the carbon-carbon backbone cleavage of aliphatic compounds. This work provides a fundamental knowledge of the biodegradation process of PE or PS at the atomic level and facilitates predicting the pathway of plastics' biodegradation by microbial enzymes.

Keywords: polyethylene, oxidation, carbon-carbon bond cleavage, quantum mechanism, bond dissociation energy (BDE)

\section{INTRODUCTION}

Plastics are widely used in industrial and household applications because of their low weight, durability and low production cost (Andrady, 2015). However, the growing amount of plastic solid waste (PSW) is a global concern. The widespread use of plastics, the lack of waste management and casual social behavior, however, pose a major threat to the environment (Leja and Lewandowicz, 2010). Despite increasing efforts to reduce the residual amounts of PSW to be disposed off through segregated collection and recycling, a considerable amount of PSW is still landfilled and the extent of PSW ocean pollution has become a worldwide issue (Baeyens et al., 2010; Brems et al., 2012).

Considering their abundance of plastics in the environment, biodegradation of plastics could be the most effective way. Decades ago, several biodegradable aliphatic polyesters such as PLA and 
$\mathrm{PHB}$, were produced to replace petrochemical plastics (Tokiwa et al., 2009). However, the most commonly used plastics are still synthetic polymers obtained from petrochemical hydrocarbons and derivatives (Geyer et al., 2017). Polyethylene (PE) and polystyrene (PS) are amongst the most important mass-produced plastics and largely manufactured into short-life products including packaging materials for food and disposable dishware (Plastics Europe, 2018). PE and PS are highly stable polymers and notably resistant to biodegradation (Ho et al., 2017). The carboncarbon backbone in PE and PS is highly resistant to enzymatic cleavage by oxidation-reduction (Goldman, 2010). Additionally, the high molecular weight and strong hydrophobic character hamper their biodegradation (Albertsson and Karlsson, 1993).

Recently, several microbes and microbial enzymes have been shown able to catalyze the degradation of various petrochemical plastics including PE and PS (Wei and Zimmermann, 2017). Shimpi et al. (2012) reported the biodegradation of modified PS by using a pure strain of Pseudomonas aeruginosa. Motta et al. (2009) used the Curvularia species to investigate the degradation of atactic PS. These results suggested that the biodegradation of PS material through using selected microbial strains might become a feasible solution for reducing the huge amount of waste and disposed plastics.

Sivan et al. isolated the actinomycete Rhodococcus ruber (C208) to degrade PE and PS (Mor and Sivan, 2008; Santo et al., 2013), and demonstrated that laccase, a copperbinding enzyme, played a crucial role in the oxidation and degradation of PE by $R$. ruber (Santo et al., 2013). In addition to laccase, several oxidoreductases were shown to be involved in the biodegradation of PE and PS, such as the AlkB family hydroxylases and hydroquinone peroxidase (Nakamiya et al., 1997; Jeon and Kim, 2015).

The catalytic mechanism of oxidoreductases with respect to the cleavage of PE and PS still remains unexplained. The present work applied quantum mechanism calculations to unveil the bio-catalytic mechanism of PE and PS degradation by oxidoreductase, with the $\mathrm{P} 450$ monooxygenase catalyzed reaction being treated as a typical saturated carbon-carbon bone cleavage reaction (Matthews et al., 2017). This work attempts to provide fundamental insights into the possible biodegradation of plastics with a C-C backbone.

\section{MATERIALS AND METHODS}

\section{Computation Methods}

In this work, geometry optimizations, relaxed scan and natural population analysis (NPA) charges were calculated by the Gaussian 09 software package (Frisch et al., 2013) at the B3LYP/6-31+G(d,p) theoretical level. The frequency of structures was also calculated at same level to ensure that the stable structures have no imaginary frequency, and only one imaginary frequency for the transition state. Fuzzy bond orders, spin density and spin population analysis were calculated by Multiwfn (Lu and Chen, 2012). Relaxed force constants were calculated by Compliance (Brandhorst and Grunenberg, 2008; Brandhorst and Grunenberg, 2010).

\section{Analysis of Three Bond Strength Descriptors}

The bond dissociation energy (BDE), widely used in the literature as a kind of bond strength descriptor can be defined as the standard enthalpy $(\mathrm{H})$ change when a bond is cleaved by homolysis to produce two fragments. In some cases, it is however better to calculate the standard enthalpy change when a bond is cleaved by heterolysis. These calculations of Bond Dissociation Energy (BDE, at 298K) are shown in the following equation:

$$
B D E(298 \mathrm{~K})=\mathrm{H}\left(\mathrm{RH}_{2} \bullet\right)+\mathrm{H}(\bullet \mathrm{COOH})-\mathrm{H}\left(\mathrm{RCH}_{2}-\mathrm{COOH}\right)
$$

However, the numerical value of BDE, as an intrinsic strength of a particular bond, depends on the stable molecule and the stability of the fragments, such as electronic ground state, minimum conformation, etc. (Grunenberg, 2017). The bond order is a quantitative description of chemical bonds and has been widely used by chemists to understand the nature of molecular electronic structure and predict the molecular reactivity, aromaticity, and stability (Lu and Chen, 2013). The fuzzy bond order exhibits very little basis set sensitivity and will not be deteriorated by using diffuse basis functions (Mayer and Salvador, 2004). For the same type of bonds, the fuzzy bond order positively correlates to bond strength.

Force constants are widely used as an intuitive bond strength descriptor. However, the numerical values of rigid force constants depend on the choice of coordinate systems. In order to overcome this disadvantage and achieve a higher precision, the compliance matrix, which is a second-order tensor containing non-zero coupling elements (Grunenberg, 2017), was adopted in this work to describe the bond strength.

\section{RESULTS AND DISCUSSION}

\section{Model Structures and Charge Distribution Analysis}

In this work, the structures of Figure 1 were investigated to determine the cleavage of the $\mathrm{C}-\mathrm{C}$ bond close to the carboxyl group under both acidic or alkaline conditions. Since the four molecules are very similar, spin density and NPA charges were calculated to ensure that these structures are reliable. The bond length of the $\mathrm{C} 2-\mathrm{C} 3$ bonds (C3 is the alpha carbon, $\mathrm{C} 2$ is the carboxylic carbon atom) in the molecules and the NPA charges of certain atoms are given in Table 1. It is specifically mentioned here that for the structure shown in Figures 1A,E, a stable configuration is not obtained after a plenty of structural optimizations. The C2-C3 bond cleavage occurred in every optimization attempt with molecule Figure $\mathbf{1 E}$ as the initial structure. Therefore, structures with optimization are adopted as the basis for this part of the study.

The results of the spin density in Figure 1 show that there are no unpaired electrons in the ionic structures, while some unpaired electrons exist in the free radical structures mainly in O1, O4, and C5. As shown in Table 1, NPA charges of the C5s in ionic structures for both an alkaline and an acidic environment are positive, while the NPA charges are negative for the two other 
A<smiles>[R]CC([CH2+])CC(=O)O</smiles>

C<smiles>[R]C[CH+]CC(=O)O</smiles>

E

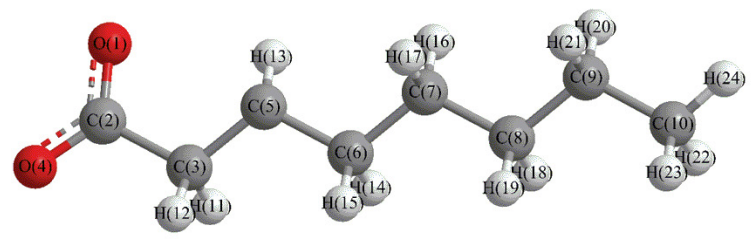

G

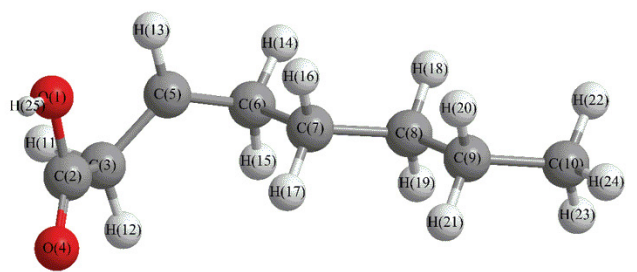

B<smiles>[R]CC([2H])CC(=O)[O-]</smiles>

D<smiles>[R]CC([2H])CC(=O)O</smiles>

$\mathbf{F}$

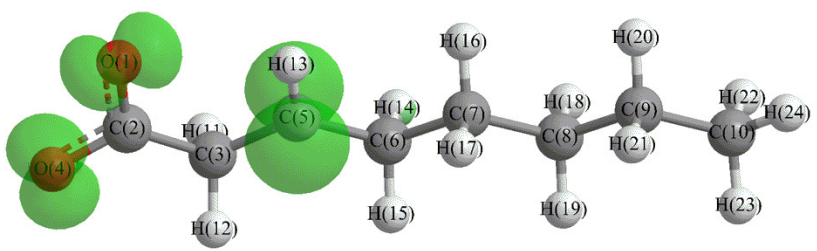

H

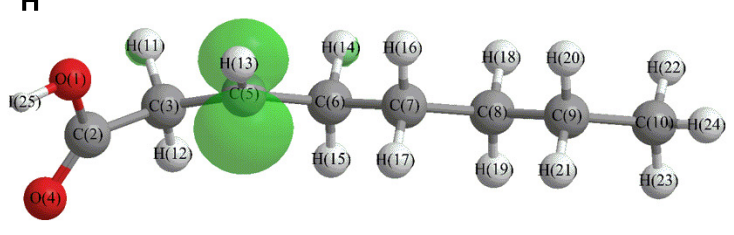

FIGURE 1 | Molecular structure: (A) lonic structure in alkaline condition; (B) Free radical structure in alkaline condition; (C) lonic structure in acidic condition; (D) Free radical structure in acidic condition. (E-H) are corresponding spin density for molecules (A-D), which was marked with green. The value of the iso-surface is 0.01. The carbon atoms, C2 is the carboxylic carbon atom, C3 is the alpha carbon.

TABLE 1 | Bond lengths of C2-C3 bonds and certain atomic NPA charges.

\begin{tabular}{|c|c|c|c|c|}
\hline molecule & $\begin{array}{c}\text { bond lengths } \\
\text { of C2-C3 } \\
\text { bonds/ } \AA\end{array}$ & $\begin{array}{c}\text { NPA } \\
\text { charges of } \\
\text { C5/a.u. }\end{array}$ & $\begin{array}{c}\text { NPA } \\
\text { charges of } \\
\text { 01/a.u. }\end{array}$ & $\begin{array}{c}\text { NPA } \\
\text { charges of } \\
\text { 04/a.u. }\end{array}$ \\
\hline & 1.569 & 0.173 & -0.625 & -0.579 \\
\hline & 1.502 & -0.128 & -0.473 & 0.388 \\
\hline & 1.551 & 0.230 & -0.506 & -0.723 \\
\hline & 1.515 & -0.124 & -0.735 & -0.604 \\
\hline
\end{tabular}

structures. Compared to the NPA charges of oxygen atoms of the ionic structure, there are more negative charges in the oxygen atom $\mathrm{O} 1$ in ionic structure in alkaline environment. Therefore, these structures are appropriate for further calculations.

\section{Effect of the Structure on C-C Bond Cleavage}

\section{The Ionic Structure Under Alkaline Condition}

For structure (A) of Figure 1, no stable corresponding structure was obtained after several rounds of optimization. Therefore, an optimized free fatty acid was used as template, a hydride ion at $\mathrm{C}_{\beta}$ was removed to obtain the approximate structure for further calculation. The approximate structure is shown in Figure 1E.

Firstly, a geometry optimization was performed for this structure, and the result is shown in Figure 2A. It is obvious 
that the molecule is cleaved into a linear olefin and $\mathrm{CO}_{2}$ which are obtained from the cleavage of the fatty acid carboxylate. Then, a potential energy surface relaxed scan was performed along the distance of the $\mathrm{C} 2-\mathrm{C} 3$ bond in the molecule as shown in Figure 1E, to describe the energy of this system. The result of the scan is shown in Figure $\mathbf{2 B}$, demonstrating that the energy decreases quickly as the distance increases initially, which indicates that the $\mathrm{C} 2-\mathrm{C} 3$ bond in the molecule as shown in Figure $\mathbf{1 A}$ is very unstable.

For a more thorough study, the transition state which is shown in Figure 3A, was also calculated based upon the unstable structure as shown in Figure 1E. The vibration direction of the imaginary frequency is mainly along the direction of the C2-C3 bond stretching. A stable structure with a minimum in potential energy surface and connecting the transition state structure was calculated as shown in Figure 3B. The stable structure consists of a linear olefin and $\mathrm{CO}_{2}$ as the products derived from the cleavage of fatty acids. The energy of the molecule, as shown in Figure 1E is $110.7 \mathrm{~kJ} / \mathrm{mol}$ higher than the energy of the transition state, which confirms the high instability of the molecule as shown in Figure 1E. Through the above calculations, it is clear that the ionic structure in an alkaline environment is close to the transition state structure in its potential energy surface, which indicates the instability of the $\mathrm{C} 2-\mathrm{C} 3$ bond.

\section{The Free Radical Structure Under Alkaline Condition}

Based upon the free radical structure in an alkaline environment as shown in Figure 1F, a transition state optimization was carried out. The change of spin multiplicity caused by the cleavages of $\mathrm{C} 2$ and C3 did not consider here. The distance of C2-C3 in this transition structure is $2.23 \AA$, which means that this molecule is divided into two parts. Table 2 and Figure 4 show the result of the spin population analysis which was carried out to determine the distribution of single electrons in the transition structure. This analysis shows that more than $80 \%$ (about two electrons) of single electrons are on the part of the free carboxyl group, indicating that a linear olefin was produced. Assessing the normal mode corresponding to the imaginary frequency, it was found that the composing displacements tend to lead in the directions of the structure derived from the cleavage of the fatty acid carboxylate. Therefore, the transition state structure connects the product consisting of a linear olefin and $\mathrm{CO}_{2}$. The energy of the transition state includes zero-point correction and is $109.8 \mathrm{~kJ} / \mathrm{mol}$ relative to the molecule as shown in Figure 1F. Due to the high energy of the transition state, it is not easy to cleave the bond in this structure.
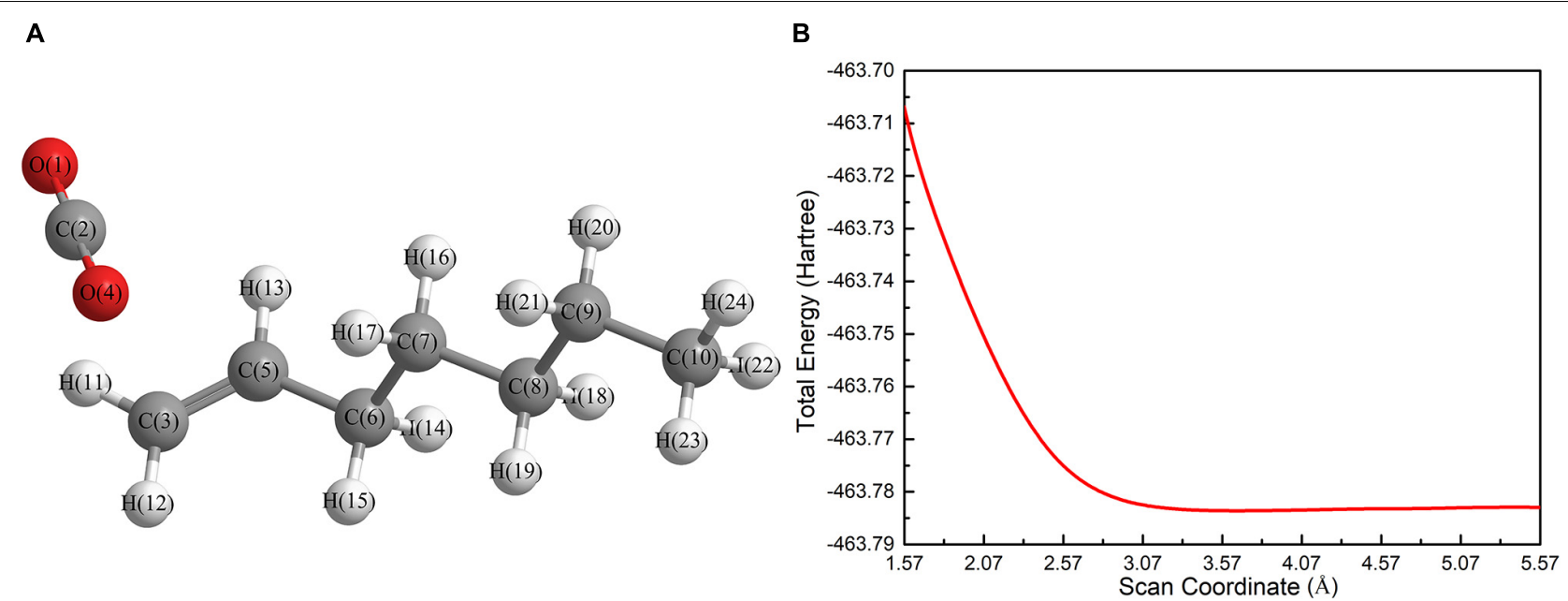

FIGURE 2 | (A) lonic structure in alkaline condition after optimization; (B) the result of relaxed scan along the distance of the C2-C3 bond based upon the unstable structure as shown in Figure 1E. The unit in (B) is Hartree, $1 \mathrm{Ha}=2625.499638(65) \mathrm{kJ} / \mathrm{mol}$.

A

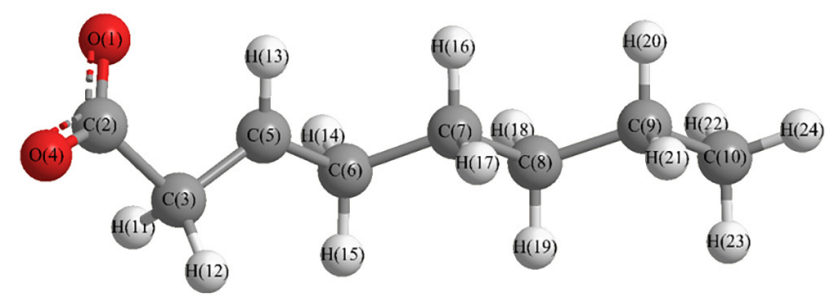

B

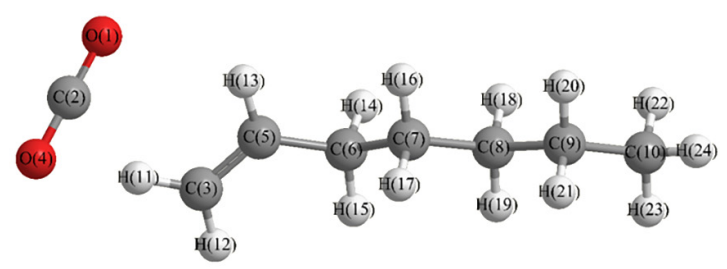

FIGURE 3 | (A) Optimized transition state structure, based upon the unstable structure as shown in Figure 1E; (B) One stable structure connects the transition state structure. 
TABLE 2 | Result of spin population analysis based on the transition structure shown in Figure 4.

\begin{tabular}{lrrr}
\hline Atomic space & Value & \% of sum & \% of sum abs \\
\hline O1 & 0.64107952 & 32.054013 & 30.046278 \\
C2 & 0.42337472 & 21.168761 & 19.842834 \\
C3 & -0.06553244 & -3.276626 & -3.071391 \\
O4 & 0.64994558 & 32.497317 & 30.461815 \\
C5 & 0.30392666 & 15.196351 & 14.244512 \\
\hline
\end{tabular}

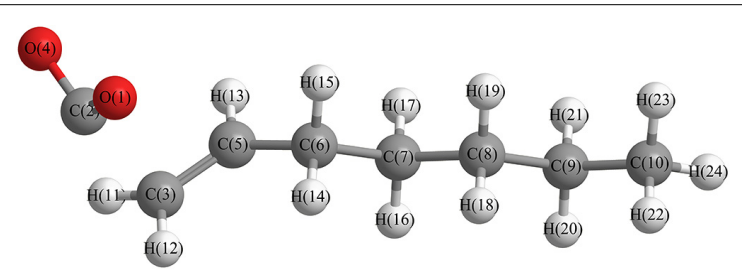

FIGURE 4 | Optimized transition structure, based upon the free radical structure in alkaline condition.

TABLE 3 | Bond orders, relaxed force constants and BDEs of the C2-C3 bonds in the selected molecules.

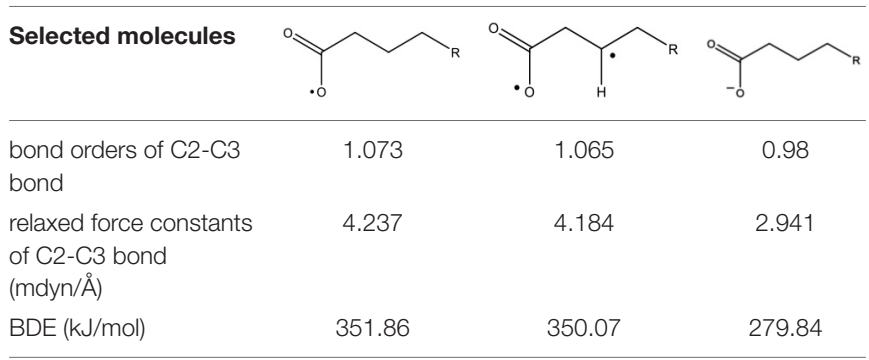

As can be seen from Table 3, when a hydrogen atom has been removed from $\mathrm{C}_{\beta}$, the changes of BDEs, relaxed force constants and the bond orders are very small. So the $\mathrm{C} 2-\mathrm{C} 3$ bond strengths in both structures are very similar and the lack of a hydrogen atom at the location of $\mathrm{C}_{\beta}$ has little influence on the C2-C3 bond strength.

By comparing the third column with the fourth column in Table 3, it is obvious that the bond order, relaxed force and $\mathrm{BDE}$ of the $\mathrm{C} 2-\mathrm{C} 3$ bond in the free radical structure in an alkaline environment are much higher than the C2C3 bond in a carboxylate anion, which indicates that the $\mathrm{C} 2-\mathrm{C} 3$ bond strength of the free radical structure in an alkaline environment is stronger. Because decarboxylation is less favorable at low temperatures and highly sensitive to conditions for carboxylic acids, decarboxylation of the molecule in Figure 1F is more difficult.

\section{Ionic Structure Under Acidic Condition}

If there is a linear olefin produced, the $\mathrm{C} 2-\mathrm{C} 3$ bond in this structure should be cleaved by heterolysis, and hence Table 4 gives the heterolytic bond dissociation energies of the $\mathrm{C} 2-\mathrm{C} 3$ bonds. By comparing the second and the fourth columns in Table 4, it can be seen that the values of the bond order, relaxed force constant, BDE and heterolytic bond dissociation energy of the $\mathrm{C} 2-\mathrm{C} 3$ bond become much smaller when a hydrogen anion is removed from $\mathrm{C}_{\beta}$ in a carboxylic acid, which indicates that the bond strength becomes much weaker. Comparing the fourth column in Table 4 to the fourth column in Table 3, it can be seen that the values of the bond orders, the relaxed force constants and the BDEs of the $\mathrm{C} 2-\mathrm{C} 3$ bonds are very close, so the $\mathrm{C} 2-\mathrm{C} 3$ bond strengths in the two structures are basically the same.

As the absence of a hydrogen anion in a carboxylic acid may cause the $\mathrm{O}-\mathrm{H}$ bond to become weaker, it was decided to investigate the $\mathrm{O}-\mathrm{H}$ bond strength of the ionic structure in an acidic environment and determine whether it has an impact on the decarboxylation. As can been seen from Table 4, when a hydrogen anion is removed from $\mathrm{C}_{\beta}$ in a carboxylic acid, the values of the bond order and the relaxed force constant of the $\mathrm{O}-\mathrm{H}$ bond decrease slightly and the heterolytic bond dissociation energies of the $\mathrm{O}-\mathrm{H}$ bond become much smaller. The reason why the heterolytic bond dissociation energy varies significantly may be due to the major configuration changes from the segmentation optimization calculation (C-C bond cleavage), which affects the calculation of the heterolytic bond dissociation energy. In summary, the absence of a hydride ion does not significantly affect the strength of the $\mathrm{O}-\mathrm{H}$ bond and therefore does not affect $\mathrm{C}-\mathrm{C}$ bond cleavage.

TABLE 4 | Bond orders, relaxed force constants, BDEs and heterolytic bond dissociation energies of the $\mathrm{C} 2-\mathrm{C} 3 / \mathrm{O}-\mathrm{H}$ bonds in the structures of selected molecules.

\begin{tabular}{|c|c|c|c|c|}
\hline Selected molecules & $\mathrm{OH}$ & & $\mathrm{OH}$ & $Y_{H}^{+}$ \\
\hline Bonds & C2-C3 & O-H & $\mathrm{C} 2-\mathrm{C} 3$ & O-H \\
\hline $\begin{array}{l}\text { bond order of } \mathrm{C} 2-\mathrm{C} 3 / \mathrm{O}-\mathrm{H} \\
\text { bond }\end{array}$ & 1.039 & 0.833 & 0.965 & 0.807 \\
\hline $\begin{array}{l}\text { relaxed force constant of } \\
\mathrm{C} 2-\mathrm{C} 3 / \mathrm{O}-\mathrm{H} \text { bond(mdyn/Å) }\end{array}$ & 4.115 & 7.813 & 2.915 & 7.692 \\
\hline $\mathrm{BDE}(\mathrm{kJ} / \mathrm{mol})$ & 363.32 & - & 269.58 & - \\
\hline $\begin{array}{l}\text { heterolytic bond } \\
\text { dissociation energy }(\mathrm{kJ} / \mathrm{mol})\end{array}$ & 1173.24 & 1437.5 & 216.08 & 748.33 \\
\hline
\end{tabular}

TABLE 5 | Bond orders, relaxed force constants, BDEs and heterolytic bond dissociation energies of the $\mathrm{C} 2-\mathrm{C} 3 / \mathrm{O}-\mathrm{H}$ bonds in the structures of selected molecules.

\begin{tabular}{|c|c|c|c|c|}
\hline \multicolumn{5}{|l|}{ Selected molecules } \\
\hline Bonds & $\mathrm{C} 2-\mathrm{C} 3$ & $\mathrm{O}-\mathrm{H}$ & C2-C3 & $\mathrm{O}-\mathrm{H}$ \\
\hline $\begin{array}{l}\text { bond order of } \mathrm{C} 2-\mathrm{C} 3 / \mathrm{O}-\mathrm{H} \\
\text { bond }\end{array}$ & 1.039 & 0.833 & 1.033 & 0.831 \\
\hline $\begin{array}{l}\text { relaxed force constant of } \\
\text { C2-C3/O-H bond(mdyn/Å) }\end{array}$ & 4.115 & 7.813 & 3.968 & 7.813 \\
\hline $\mathrm{BDE}(\mathrm{kJ} / \mathrm{mol})$ & 363.32 & - & 361.13 & - \\
\hline heterolytic bond & - & 1437.50 & - & 1422.34 \\
\hline
\end{tabular}




\section{Free Radical Structure Under Acidic Condition}

Table 5 shows that the bond order, relaxed force constant, and BDE of C2-C3 bond are not significantly changed in the absence of a hydride atom at $\mathrm{C}_{\beta}$ position. Moreover, the bond order, relaxed force constant, and $\mathrm{BDE}$ of this structure are much larger than the corresponding value of molecule in the fourth column of Table 3. Therefore, the bond strength of $\mathrm{C} 2-\mathrm{C} 3$ bond in this structure is very high. Table 5 also shows that the bond order, relaxed force constant, heterolytic bond dissociation energy of $\mathrm{O}-\mathrm{H}$ bond do not change significantly, in the absence of a hydride atom at $\mathrm{C}_{\beta}$ position, indicating that the bond strength of the $\mathrm{O}-\mathrm{H}$ bond does not change significantly, and hence does not affect $\mathrm{C}-\mathrm{C}$ bond cleavage.

In summary, in the ionic structure, the absence of a hydrogen anion at the $C_{\beta}$ position significantly reduces the bond strength of C2-C3. Therefore, whether in an acidic environment or an alkaline environment, $\mathrm{C}-\mathrm{C}$ bond cleavage is more likely to occur in the ionic structures. On the other hand, in an alkaline environment, the $\mathrm{C} 2-\mathrm{C} 3$ bond is more unstable because of the presence of a carboxylate anion that promotes the tendency of push electrons. Therefore, the ionic structure under alkaline conditions is most advantageous for the removal of carboxyl groups.

\section{CONCLUSION}

The enzymatic cleavage of $\mathrm{C}-\mathrm{C}$ bond in aliphatic compounds by quantum mechanism calculation was investigated. Under

\section{REFERENCES}

Albertsson, A. C., and Karlsson, S. (1993). Aspects of biodeterioration of inert and degradable polymers. Int. Biodeterior. Biodegradation 31, 161-170. doi: 10.1016/0964-8305(93)90002-J

Andrady, A. L. (2015). Plastic Products: Plastics and Environmental Sustainability. Hoboken, NJ: John Wiley \& Sons, 83-119. doi: 10.1002/9781119009405.ch4

Baeyens, J., Brems, A., and Dewil, R. (2010). Recovery and recycling of postconsumer waste materials. Part 2. Target wastes (glass beverage bottles, plastics, scrap metal and steel cans, end-of-life tyres, batteries and household hazardous waste). Int. J. Sustain. Eng 3, 232-245. doi: 10.1080/19397038.2010.507885

Brandhorst, K., and Grunenberg, J. (2008). How strong is it? The interpretation of force and compliance constants as bond strength descriptors. Chem. Soc. Rev. 37, 1558-1567. doi: 10.1039/b717781j

Brandhorst, K., and Grunenberg, J. (2010). Efficient computation of compliance matrices in redundant internal coordinates from Cartesian Hessians for nonstationary points. J. Chem. Phys. 132:184101. doi: 10.1063/1.3413528

Brems, A., Baeyens, J., and Dewil, R. (2012). Recycling and recovery of postconsumer plastic solid waste in a European context. Thermal Sci. 16, 1027-1035. doi: 10.2298/TSCI120111121B

Frisch, M. J., Trucks, G. W., Schlegel, H. B., Scuseria, G. E., Robb, M. A., Cheeseman, J. R., et al. (2013). Gaussian 09, Revision D.01. Wallingford, CT: Gaussian, Inc.

Geyer, R., Jambeck, J. R., and Law, K. L. (2017). Production, use, and fate of all plastics ever made. Sci. Adv. 3:e1700782. doi: 10.1126/sciadv.1700782

Goldman, A. S. (2010). ChemInform Abstract: organometallic chemistry: carboncarbon bonds get a break. Nature 463, 435-436. doi: 10.1038/463435a

Grunenberg, J. (2017). Ill-defined chemical concepts: the problem of quantification. Int. J. Quantum. Chem. 117:e25359. doi: 10.1002/qua.25359

Ho, B. T., Roberts, T. K., and Lucas, S. (2017). An overview on biodegradation of polystyrene and modified polystyrene: the microbial approach. Crit. Rev. Biotechnol. 38, 1-13. doi: 10.1080/07388551.2017.1355293 certain conditions, the enzyme could abstract a hydrogen anion from the aliphatic compounds, causing the absence of a hydride anion at the $\mathrm{C}_{\beta}$ position, which significantly reduces the bond strength of $\mathrm{C} 2-\mathrm{C} 3$ bond and finally results in the $\mathrm{C}-\mathrm{C}$ bond cleavage. The results reveal that oxidase or oxygenase could be involved in the $\mathrm{C}-\mathrm{C}$ bond cleavage in $\mathrm{PE} / \mathrm{PS}$, thus facilitating their biodegradation.

\section{DATA AVAILABILITY}

All datasets generated for this study are included in the manuscript and/or the supplementary files.

\section{AUTHOR CONTRIBUTIONS}

TT, MJ, HX, and LL conceived and designed the experiments. JX and ZC carried out the experiments. JX, ZC, KN, and HC analyzed the data. All authors wrote the manuscript.

\section{FUNDING}

This study was funded by the National Natural Science Foundation of China (Grant Number 21676016, 21861132017, 21676015, and 21838001), and the Fundamental Research Funds for the Central Universities (XK1802-8).

Jeon, H. J., and Kim, M. N. (2015). Functional analysis of alkane hydroxylase system derived from Pseudomonas aeruginosa E7 for low molecular weight polyethylene biodegradation. Int. Biodeterior. Biodegradation 103, 141-146. doi: 10.1016/j.ibiod.2015.04.024

Leja, K., and Lewandowicz, G. (2010). Polymer biodegradation and biodegradable polymers - a review. Pol. J. Environ. Stud. 19, 255-266.

Lu, T., and Chen, F. (2012). Multiwfn: a multifunctional wavefunction analyzer. J. Comput. Chem. 33, 580-592. doi: 10.1002/jcc.22885

Lu, T., and Chen, F. (2013). Bond order analysis based on laplacian of electron density in fuzzy overlap space. J. Phys. Chem. A 117, 3100-3108. doi: 10.1021/ jp4010345

Matthews, S., Belcher, J. D., Tee, K. L., Girvan, H. M., Mclean, K. J., Rigby, S. E., et al. (2017). Catalytic Determinants of Alkene Production by the Cytochrome P450 Peroxygenase OleTJE. J. Biol. Chem. 292, 5128-5143. doi: 10.1074/jbc.M116. 762336

Mayer, I., and Salvador, P. (2004). Overlap populations, bond orders and valences for 'fuzzy' atoms. Chem. Phys. Lett. 383, 368-375. doi: 10.1016/j.cplett.2003. 11.048

Mor, R., and Sivan, A. (2008). Biofilm formation and partial biodegradation of polystyrene by the actinomycete Rhodococcus ruber. Biodegradation 19, 851-858. doi: 10.1007/s10532-008-9188-0

Motta, O., Proto, A., De, C. F., De, C. F., Santoro, E., Brunetti, L., et al. (2009). Utilization of chemically oxidized polystyrene as co-substrate by filamentous fungi. Int. J. Hyg. Environ. Health 212, 61-66. doi: 10.1016/j.ijheh.2007.09.014

Nakamiya, K., Sakasita, G., Ooi, T., and Kinoshita, S. (1997). Enzymatic degradation of polystyrene by hydroquinone peroxidase of Azotobacter beijerinckii HM121. J. Biosci. Bioeng. 84, 480-482. doi: 10.1016/S0922-338X(97) 82013-2

Plastics Europe (2018). Plastics - the Facts 2018. Available at: www. plasticseurope.org retrieved in 2018.

Santo, M., Weitsman, R., and Sivan, A. (2013). The role of the copper-binding enzyme - laccase - in the biodegradation of polyethylene by the actinomycete 
Rhodococcus ruber. Int. Biodeterior. Biodegradation 84, 204-210. doi: 10.1016/ j.ibiod.2012.03.001

Shimpi, N., Mishra, S., and Kadam, M. (2012). Biodegradation of polystyrene (PS)-poly(lactic acid) (PLA) nanocomposites using Pseudomonas aeruginosa. Macromol. Res. 20, 181-187. doi: 10.1007/s13233-012-0026-1

Tokiwa, Y., Calabia, B. P., Ugwu, C. U., and Aiba, S. (2009). Biodegradability of Plastics. Int. J. Mol. Sci. 10, 3722-3742. doi: 10.3390/ijms10093722

Wei, R., and Zimmermann, W. (2017). Microbial enzymes for the recycling of recalcitrant petroleum-based plastics: How far are we? Microb. Biotechnol. 10, 1308-1322. doi: 10.1111/1751-7915.12710
Conflict of Interest Statement: The authors declare that the research was conducted in the absence of any commercial or financial relationships that could be construed as a potential conflict of interest.

Copyright (c) $2019 \mathrm{Xu}, \mathrm{Cui}, \mathrm{Nie}$, Cao, Jiang, Xu, Tan and Liu. This is an open-access article distributed under the terms of the Creative Commons Attribution License (CC BY). The use, distribution or reproduction in other forums is permitted, provided the original author(s) and the copyright owner(s) are credited and that the original publication in this journal is cited, in accordance with accepted academic practice. No use, distribution or reproduction is permitted which does not comply with these terms. 